
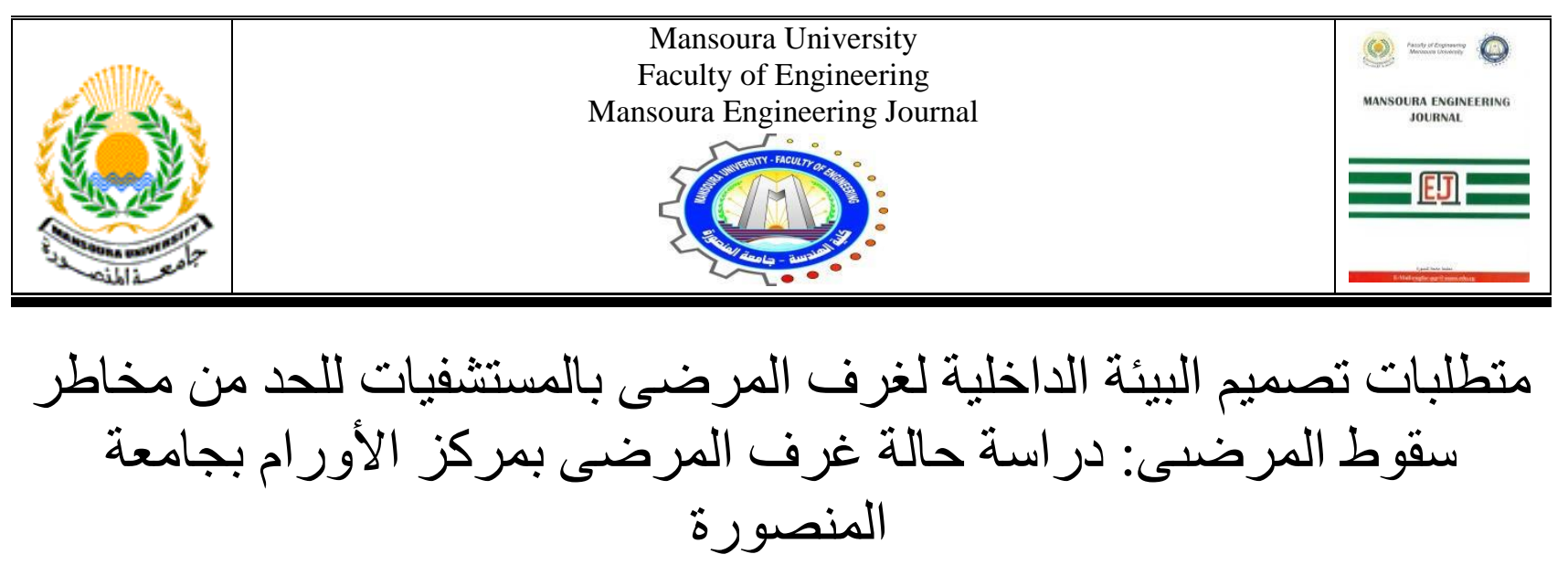

\title{
The Requirements of Internal Environment for Patients' Room Design in Hospital to Reduce the Risk of Falling the Patients. Case Study Patients' Room of Oncology Center In Mansoura University
}

Architect. Heba Mohamed Ahmed Mohamed Abdu ${ }^{1}$, Assoc. Prof. Sherif Ahmed Ali Sheta ${ }^{2}$ and Assoc. Prof. Wael Seddik Abdelatif Moustafa ${ }^{3}$

${ }^{1}$ Assistant Lecturer, Dep. of Architectural Engineering, Faculty of Engineering, Mansoura University, Email:

arch_heba84@yahoo.com

${ }^{2}$ Associate Professor in Architecture- Dep., Faculty of Engineering, Mansoura University, E-mail: shetash11@yahoo.com

${ }^{3}$ Associate Professor in Architecture- Dep., Faculty of Engineering, Mansoura University wael_seddik@yahoo.com

\begin{abstract}
The main aim of establishing cure premises is healing and recovery of illnesses and getting rid of pains. If the patient got infected with diseases or exposed to dangers inside the hospital, this is considered a demolition of the main aim of establishing this hospital because the hospital should be designed so that it can achieve the criteria of safety for patients, so we must keep a safe environment free from the dangers of patients' falling to the ground.

The main aim of the research is to identify the principles and design criteria which help reducing the risk of falling patients inside the accommodation rooms. This can be achieved by studying number of requirements that should be considered when designing such as the factors concerning organization, spatial and unit accommodation planning for bathroom inside the room, Determine the internal characteristics of the environment such as lighting and finishes that determines the noise level.

To achieve the goal the research deals with requirements of planning and design for these rooms to reduce the dangers of falling patients. This is done through a theoretical and analytical study that come to conclusions and provides recommendations on the design of patient rooms in hospitals.
\end{abstract}

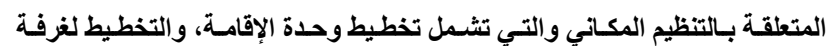

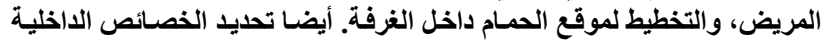
للبيئة كالإضاءة والتشطيبات وتحليد مستوي الضوضاء.

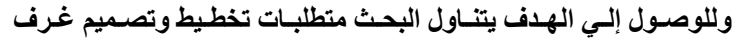

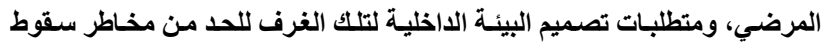

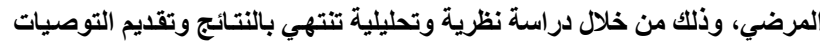

عند تصميم غرف المرضي بالمستشفيات.

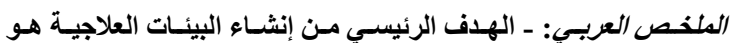

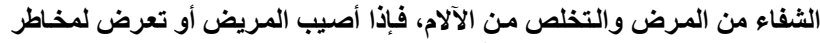

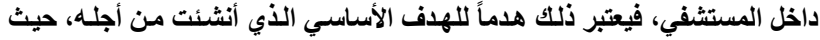

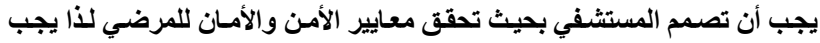

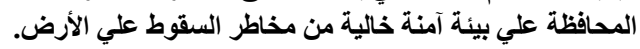

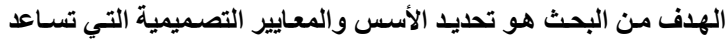

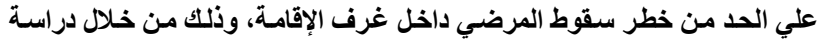

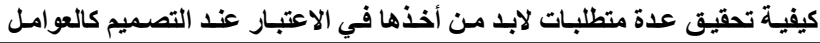


A: 20 HEBA MOHAMED AHMED MOHAMED ABDU, SHERIF AHMED ALI SHETA AND WAEL SEDDIKBDELATIF MOUSTAFA

3. الأسس التصميمية للحد من خطر سقوط المرضي.

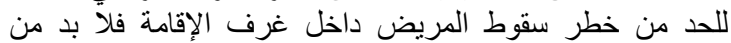

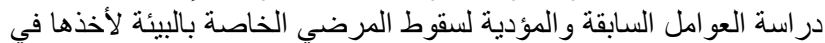

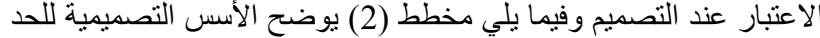

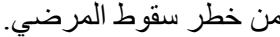

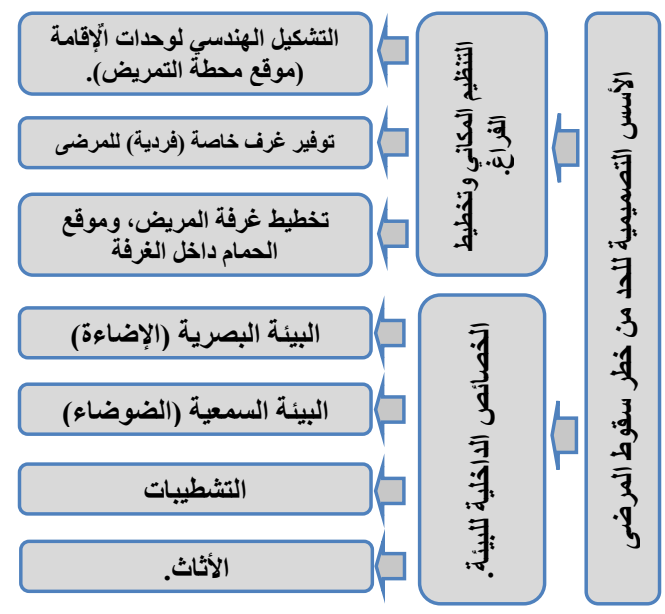

مخطط(2) الأسس التصميمية للحد من خطر سقوط المرضى المقى المصدر [1] بتصرف

1-3 التنظيم المكانسي 1-1-3

غرفة المريض هي حيث الفراغ يقضي المرضي معظم الإقامة في

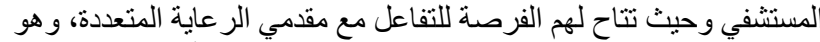

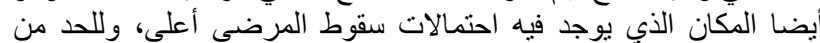

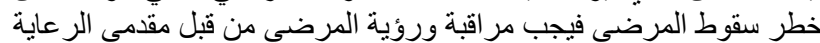

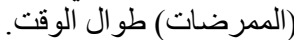
لذا فغرفة المريض يجن الن أن تثو افر فيها التالي:

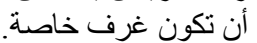

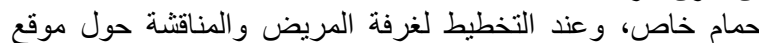

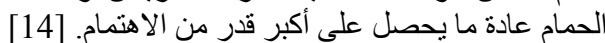

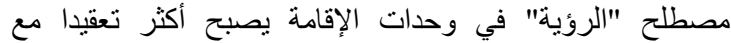

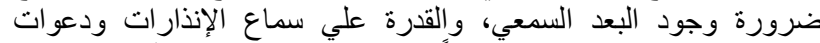

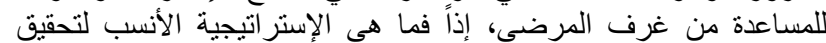

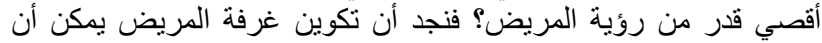

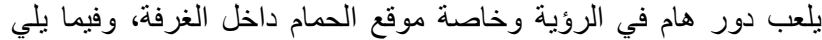
مخطط (3) يوضح التكوينات المختلفة لغرف المرف المرضي:

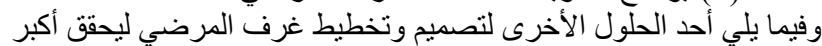

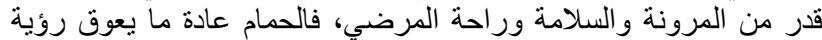
الممرضات للمرضي وفيما يلي شكل (1) يوضح المر أحد الحلول.

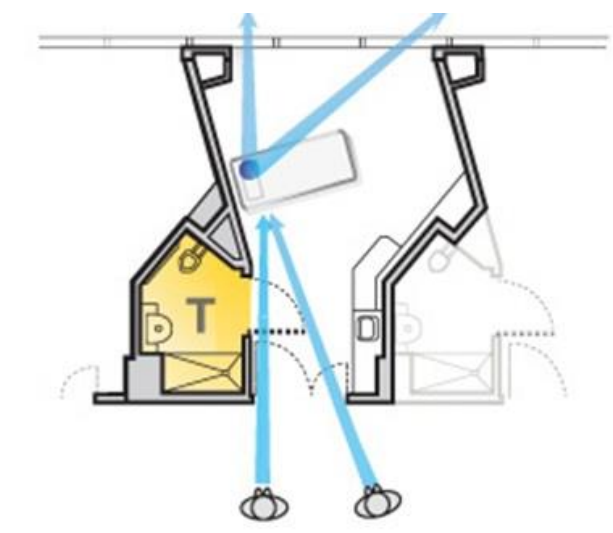

شكل (1) أحد الحلول في تصميم وتخطيط غرفة المريض لتحقيق أقصي قدر

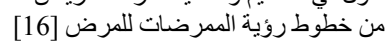

1

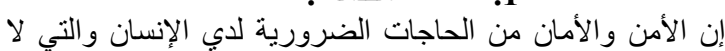

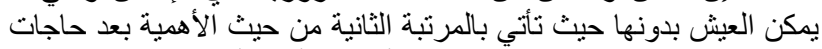

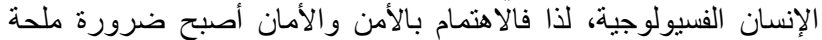

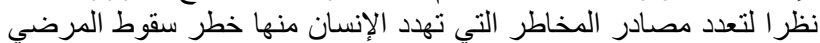

بالمستشفيات[27] بلتعدد

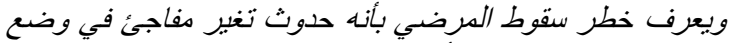

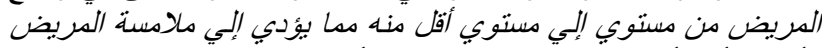

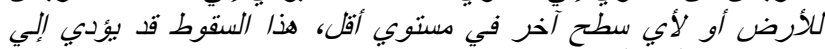

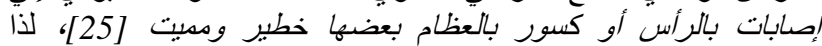

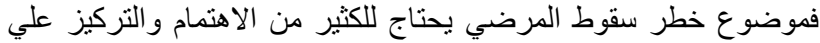
منطلبات الحد منه وتوفير ها من منطلبات تصميم المستشفي.

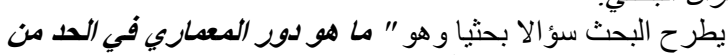
1-1 السؤال البحثي.

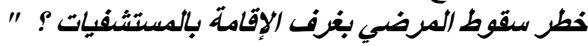

2-1 2-1 العزف من البحث.

يهدف البحث إلي تقديم طرحا خاصا للمتطلبات والأسس التصديمية لغرف المرضي للحد من خطر سقوط المرضي.

3-1 - 3 الفرضية البحثية.

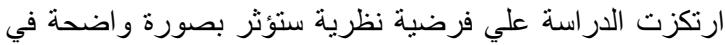

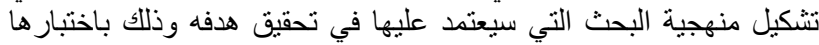

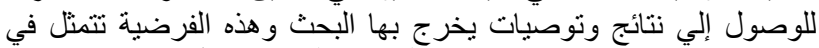

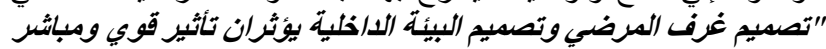
في الحد من مخاطر سقوط المرضيري" .

4-1 المنهجية البحثية.

ومن ثم فقد بني البحث منهجيته من خلال الثق النظري الذي ارتكز

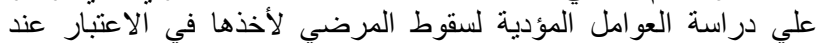

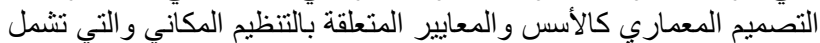

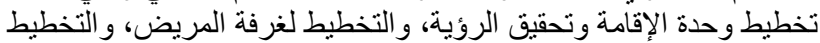

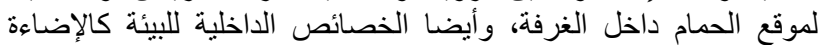

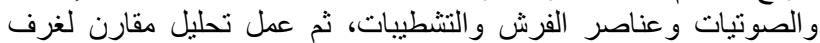
المرضي بمركز Rush University Medical بشيكاغو حيث يعد من وني

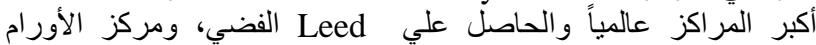
بجامعة المنصورة كمثال لنموذج محلي، وصالي وصول إلي النتائج والتوصيات

2. العوامل المؤدية لسقوط المرضي.

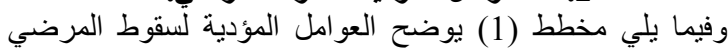
حتى بتم تفاديها.

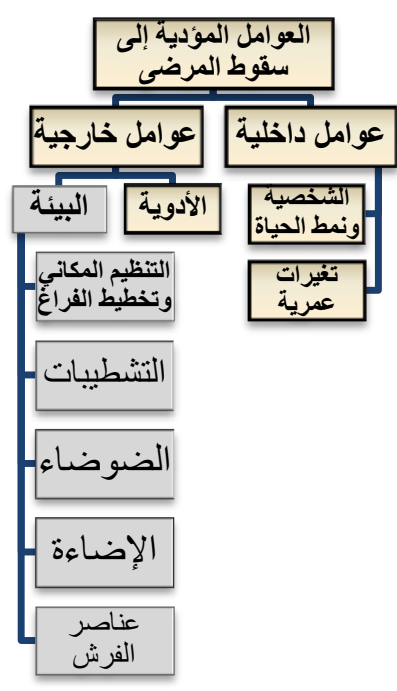




$$
\begin{aligned}
& \text { توفير غرف خاصة قد يكون عامل وقائي ضد خطر سقوط الغرف المشتركة عن الغرف الفردية [1] } \\
& \text { المريض، وأثبتت الدراسات أن خطر سقوط المريض يزداد } 4 \text { أضعاف في في فئ فئ }
\end{aligned}
$$
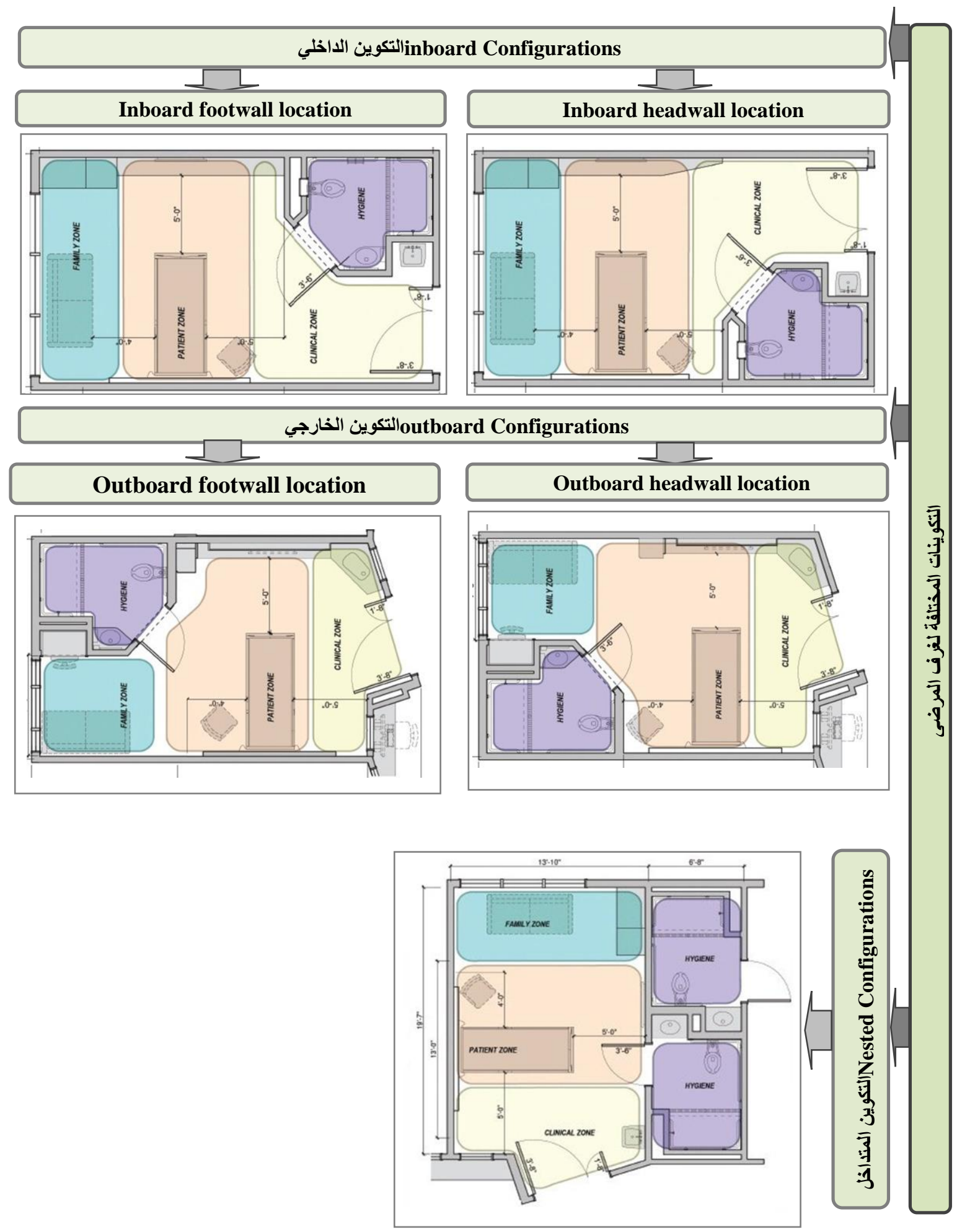


\section{A: 22 HEBA MOHAMED AHMED MOHAMED ABDU, SHERIF AHMED ALI SHETA AND WAEL SEDDIKBDELATIF MOUSTAFA}

التكوين الاخلي inboard Configurations: يقع الحمام علي جار ممر غرفة المريض.

\begin{tabular}{|c|c|}
\hline عيوبه & مميز اته \\
\hline 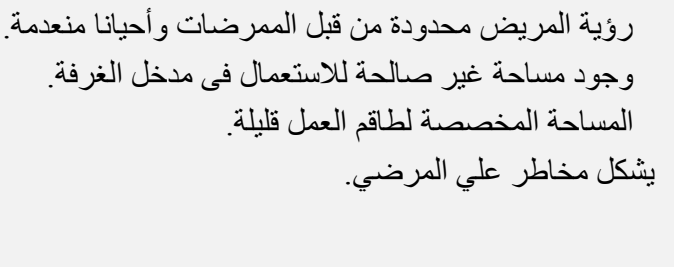 & 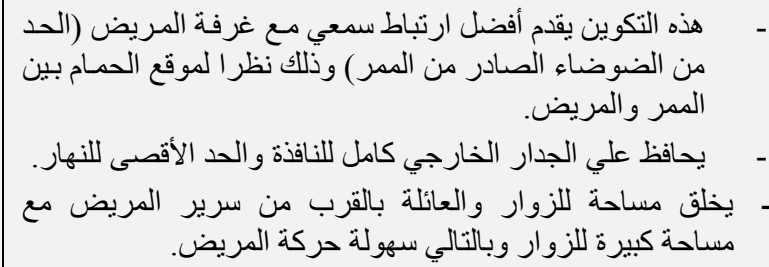 \\
\hline
\end{tabular}

التكوين الخارجي outboard Configurations: يقع الحمام علي جدار النافذة.

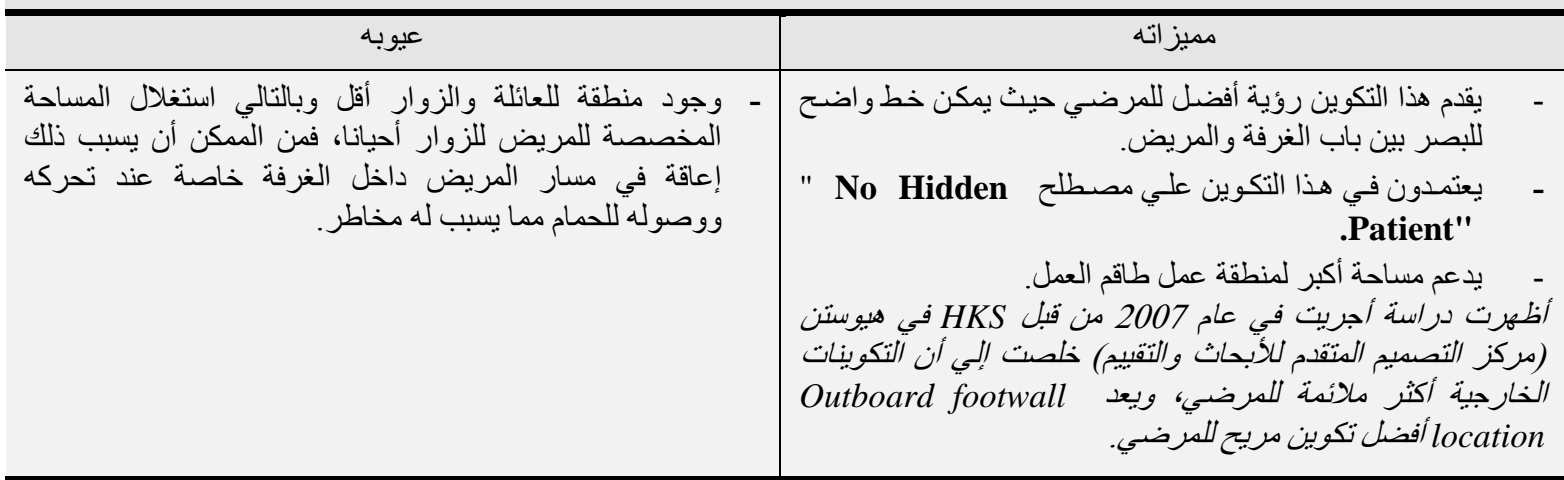

\section{Nested Configurations التكوين المتداخل}

- ـ يتجمع حمامات غرفتين متجاورتين للمشاركة في طول جدار واحد مشترك، من مميز اته الدد من السباكة وبالتالي تكلفة أقل، أيضا يوفر

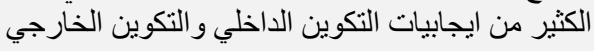

$$
\text { جدول رقم (1) مميز ات وعيوب التكوينات المختلفة لغرف المرضى (المصدر [14] [4] بتصرف ). }
$$

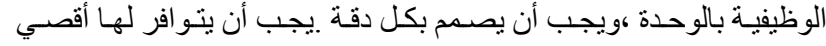

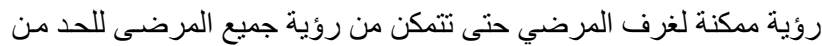

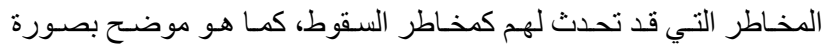

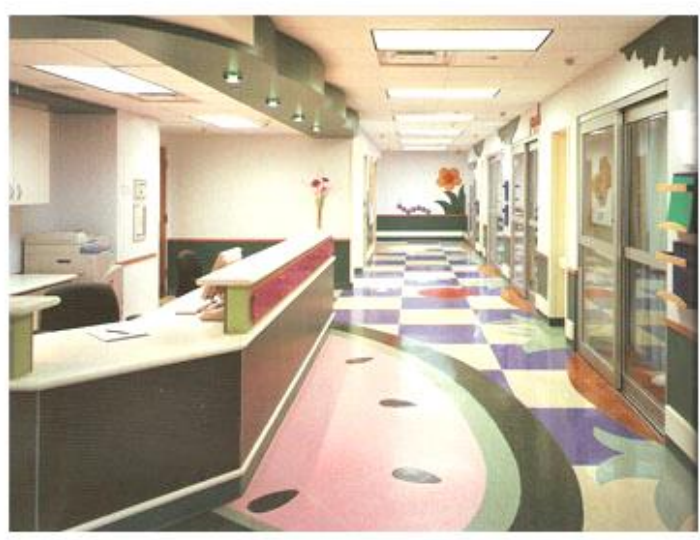

صورة (3) محطة التمريض بـ[13] Children's Memorial Hospital, Chicago, Illinois
تخطبط غرفة المريض وموقع الحمام داخل الغرفة:

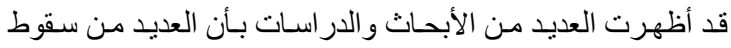

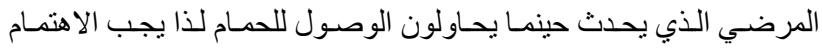

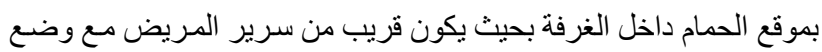

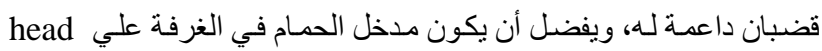

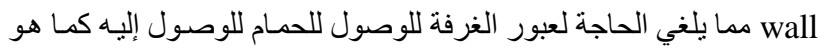

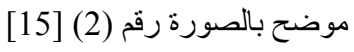

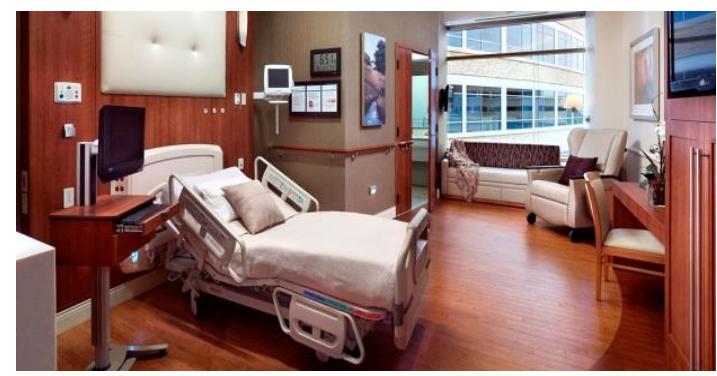

صورة (2) لغرفة المريض بمستشفي Northwestern بالولايات المتحدة

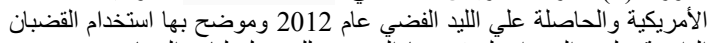

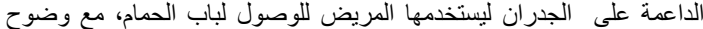

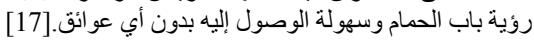

2-1-3 محطة التصريض:

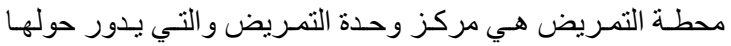
جميع أنشطة الوحدة لذا يجب أن تقع في موقع متوسط بين جميع الأنشطة ودة 
ويجب تو افر الإضـاءة الطبيعية والإضـاءة الصناعية. الإضاءة الطبيعية لغرف المباعة الطبيعي:

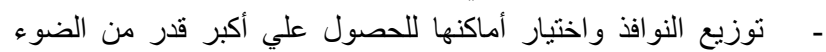

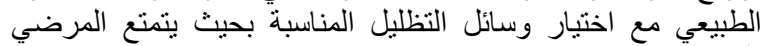
بأثنعة الثمس من دون الثئ الحصول علي الطاقة الثمسية المفرطة [2] المتوهجة الإضاءة الصناعية لغرف المرضي:

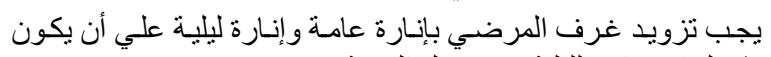
تشغيل الإضـاءة الليلية من مدخل الغرفة فئل

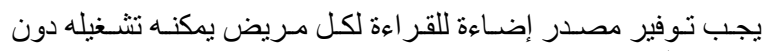
مغادرة السرير.

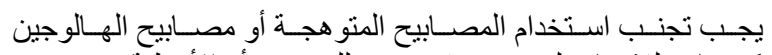

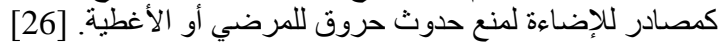

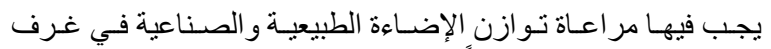

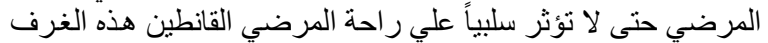

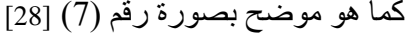

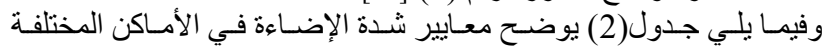
بوحدات الإقامة.

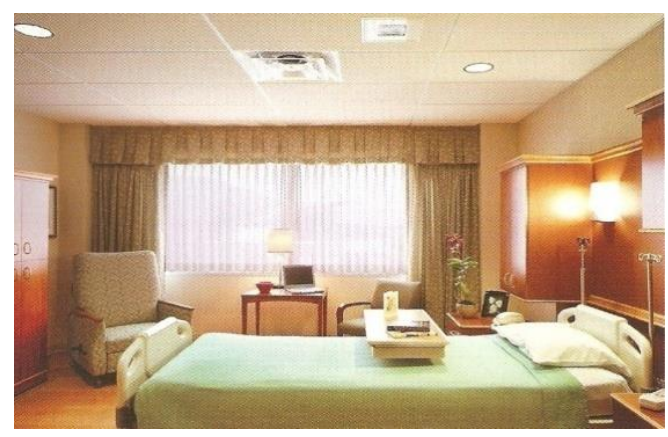

صورة (7) بمستشفى[13]

The Wisconsin Heart Hospital, Wauwatosa.

\begin{tabular}{|c|c|c|}
\hline \multicolumn{2}{|c|}{ شدة الإضاءة (لاكس) } & \multirow[t]{2}{*}{ 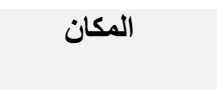 } \\
\hline الر أسية & الأفقية & \\
\hline \multicolumn{3}{|c|}{ قسم التمريض } \\
\hline 30 & 50 & عام \\
\hline 100 & 500 & الملاحظة والعلاج \\
\hline \multicolumn{3}{|c|}{ محطات التمريض مح ماض } \\
\hline 50 & 300 & عام \\
\hline 100 & 500 & منصة الاستعلام \\
\hline 30 & 50 & الطرقات نهارا \\
\hline 30 & 30 & الطرقات ليلا \\
\hline 100 & 500 & غرف الأدوية \\
\hline \multicolumn{3}{|c|}{ 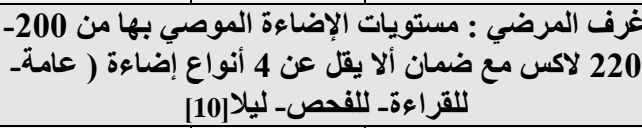 } \\
\hline 30 & 50 & عام \\
\hline 30 & 30 & الملاحظة \\
\hline 100 & 500 & الفحص الدقيق \\
\hline 50 & 300 & القراعة \\
\hline 30 & 300 & دورات المياه \\
\hline
\end{tabular}

جدول(2) معايير شدة الإضاءة في الأماكن المختلفة بوحدات الإقامة [26]

$$
\text { البيئة السدعية (الضوضاء). }
$$

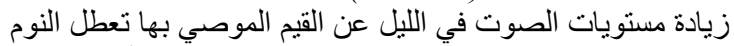

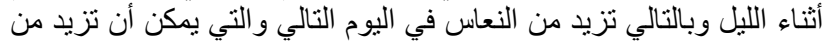

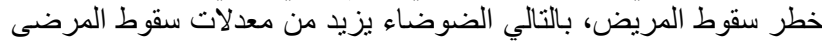

فيجب ألا يتعدي مستوي شـدة الضوضـاء داخل الأمـاكن المختلفـة

بوحدات التمريض القيم الواردة بالجدول التالي رقم (3):
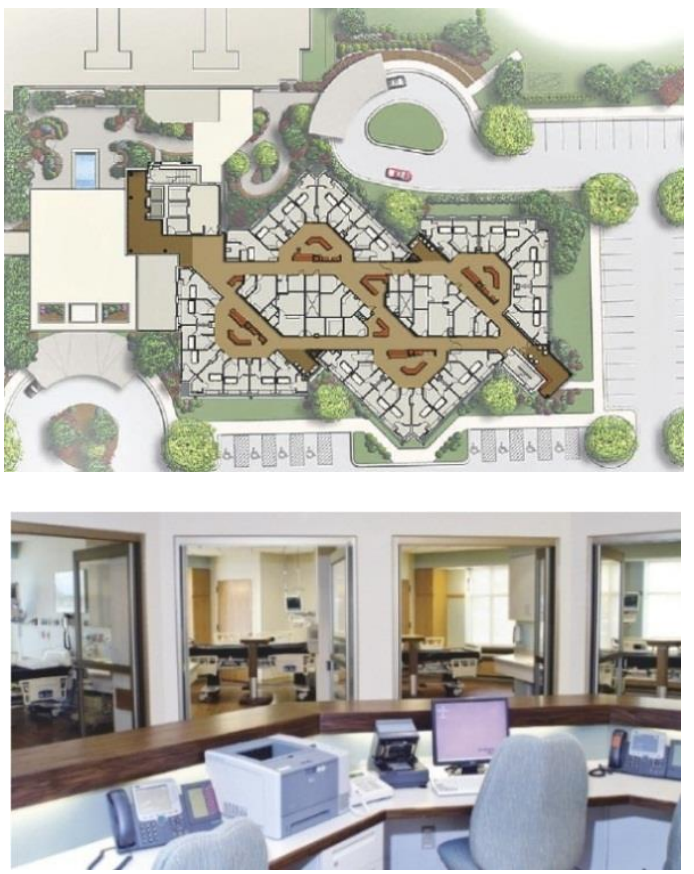

صورة (4) توزيع محطات التمريض بـ

The Heart Hospital at Swedish American

مع مر اعاة رؤية جميع أسرة المرضى [5]

3-1-3 طاولة العمل الطبية:

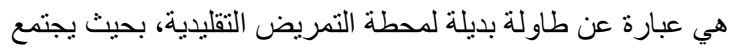

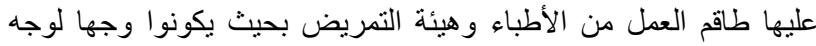

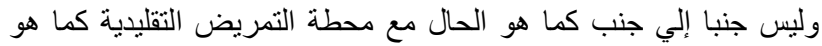

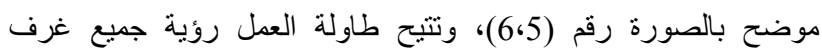

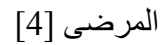

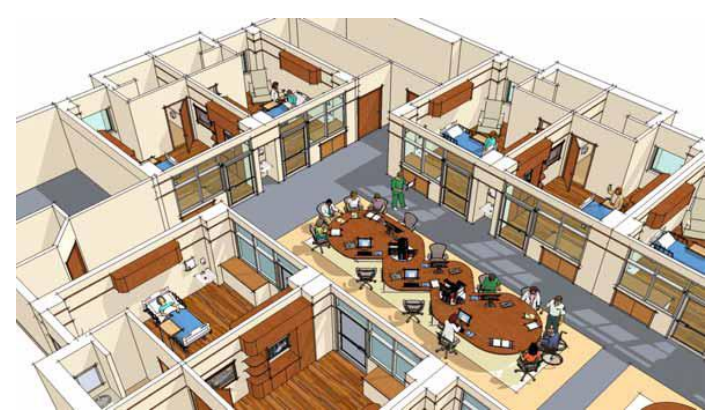

صورة (5) شكل طاولة العمل بمحطات التمريض

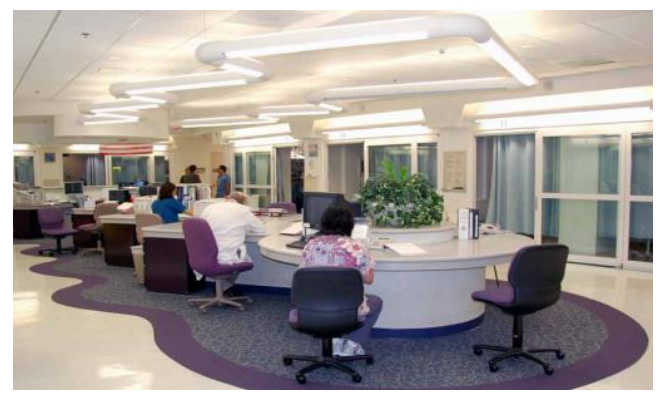

صورة (6) شكل طاولة العمل بمحطات التمريض [4]

2-3 الخصائص الداخلبة للبيئة. 1-2-3

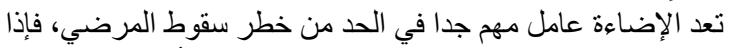

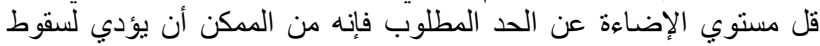

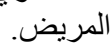


A: 24 HEBA MOHAMED AHMED MOHAMED ABDU, SHERIF AHMED ALI SHETA AND WAEL SEDDIKBDELATIF MOUSTAFA

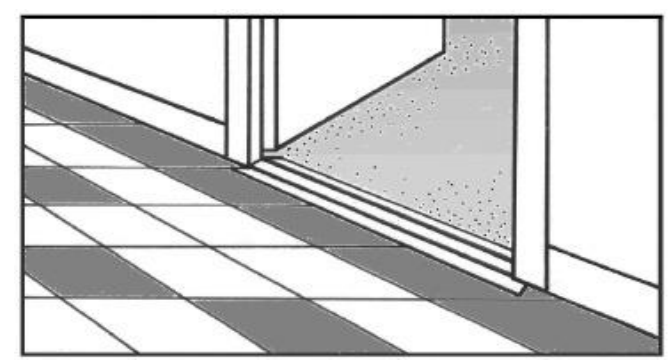

صورة (9) مثال غير جيد لفرق تشطيب أرضية فر اغين:

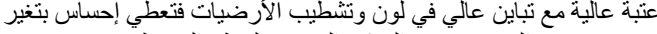
المستوي ومن الممكن التعرض لخطر السقوط.

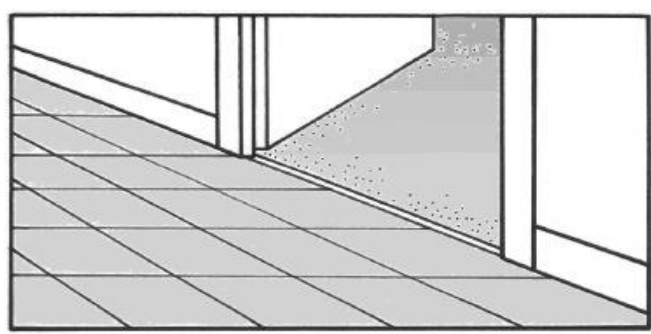

صورة (10) مثال لعملية انتقالية جيدة : تغير خفيف في الأرضيات بين الفراغين [3]

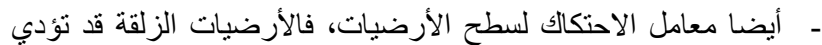

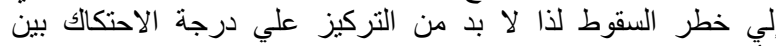

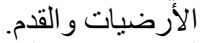

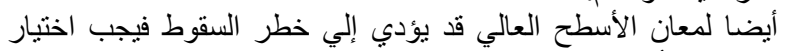

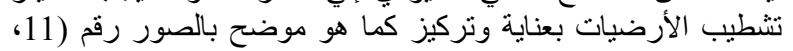

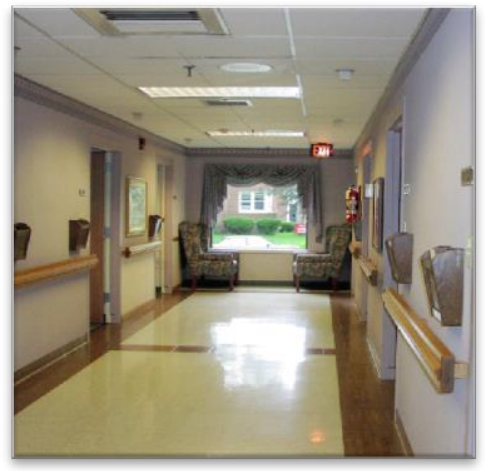

صورة (11) مثال غير جيد للمعان السطح: فضوء النافذة وضوء المصابيح

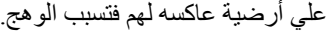

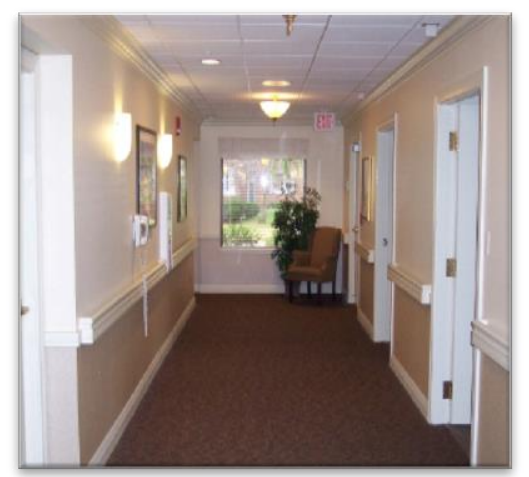

صورة (12) مثال جيد لاختيار تشطيب الأرضيات فلا يوجد أي وهج [3]

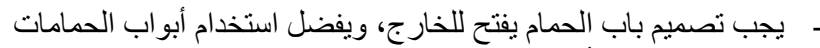

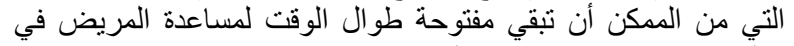

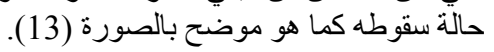

\begin{tabular}{|c|c|}
\hline $\begin{array}{c}\text { مستوي شدة الضوضاء } \\
\text { Leg ( DB(A) ) }\end{array}$ & نوع الفراغ \\
\hline $40-35$ & غرف المرضي \\
\hline $50-40$ & ممرات \\
\hline $50-40$ & الاستر احتظات وأماكن \\
\hline $55-45$ & دورات المياه \\
\hline
\end{tabular}

جدول رقم (3) مستوي شدة الضوضاء المكافئ داخل وحدات التمريض.[26].

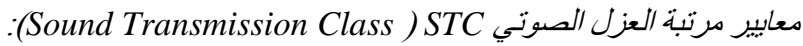
- يجب أن تحقق الحوائط مرنبة العزل الصوتي (STC45) بحيث تكون

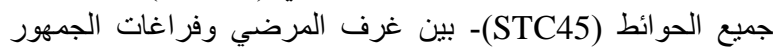

(STC45)- بين غرف المرضي وفر اغات الخدمة (STC65) (STC4).

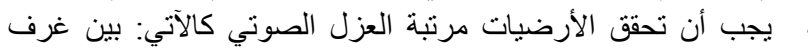
المرضي وفراغات الجمهور(

وفر اغات الخدمة، وبين غرف المرضي وضئ والطرقة أسفلها (STC 45).

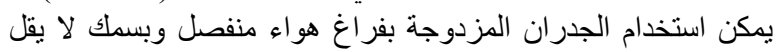

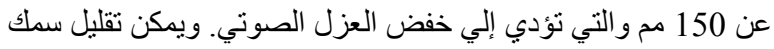

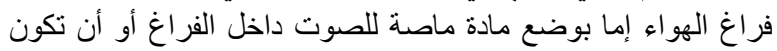

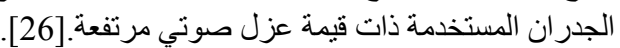

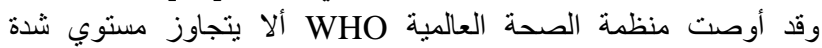

الضوضاء عن 35 ديسييل أثناء النهار، و30 ديسييل أثناء الليل. [6].

3-2-3

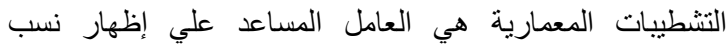

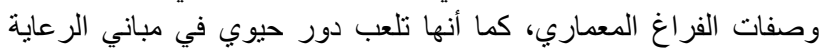

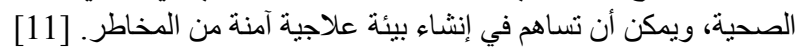

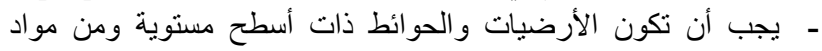
تشطيب بدون فو اصل ما أمكن.

- -

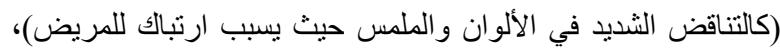

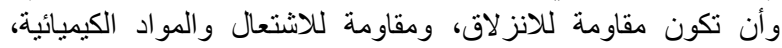

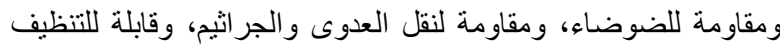

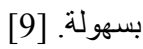
- يجب عدم استعمال السجاد أو الموكيت بغرف الإقامة لأنه أحد العوامل

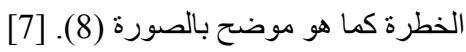

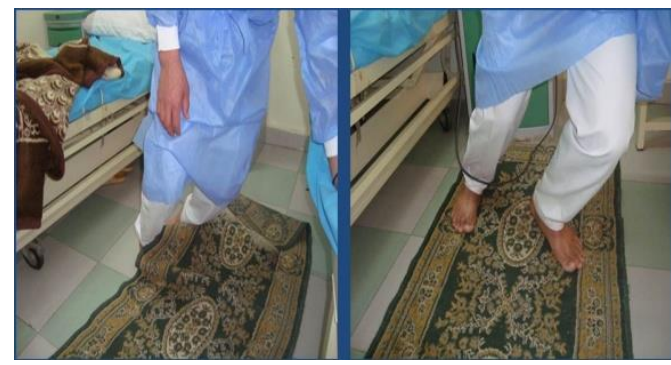

صورة (8) سقوط مريض بسبب السجادة عند نزوله من السرير [25]

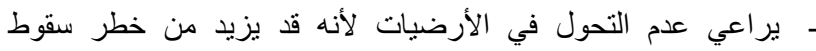

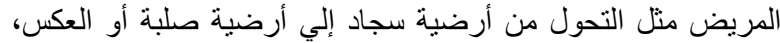

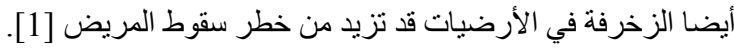

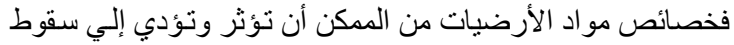

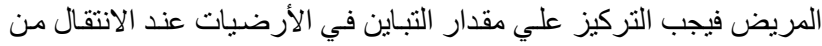

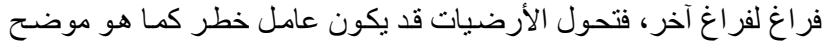

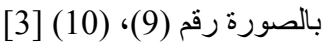




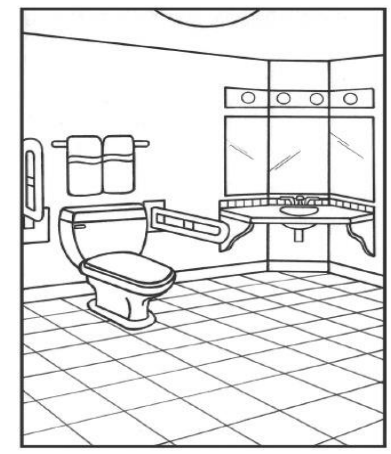

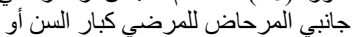
[3 [3ضي بشلل وخلافيه كرضي
صورة (16) استخدام مقابض ارتكاز علي

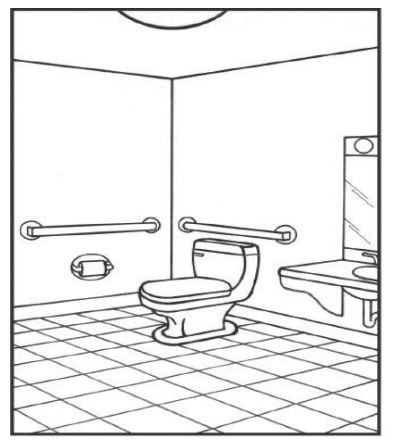

صورة (15) استخدام مقابض ارتكاز خلف وبجانب المرحاض.

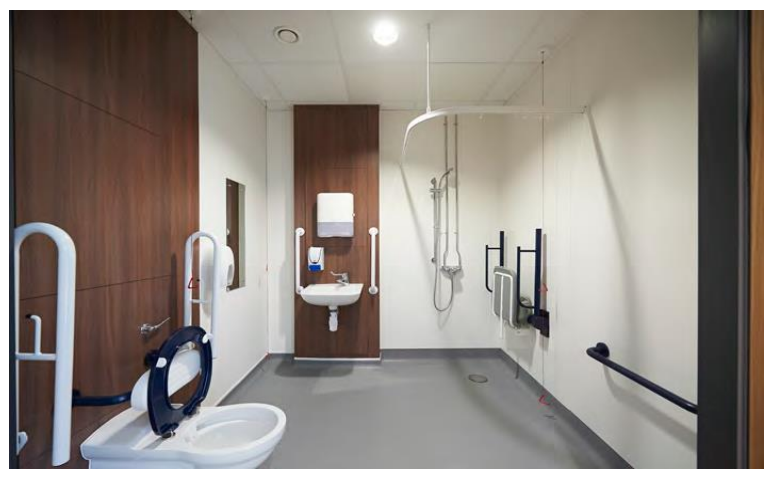

صورة (17) فراغ الحمام ب

Cocker mouth Community Hospital and Health Centre وفيه تم تصميم مقابض ارتكاز بكل عنصر بالحمام لسلامة المريض من خطر السقوط [7]

\section{4. ت تحليل مقارن.}

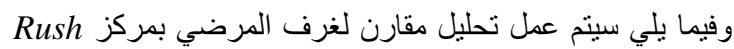
بniversity Medical

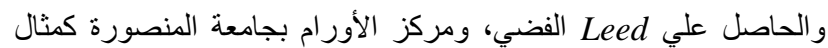
لنموذج محلي.

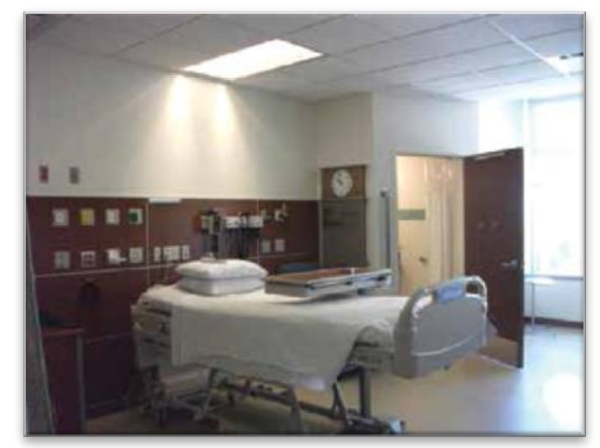

صورة (13) استخدام أبو اب للحمام من نوع تظل مفتوحة طو ال الوقت [1]

$$
\text { الأثاث (عناصر الفرش). }
$$

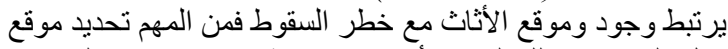

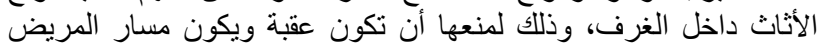

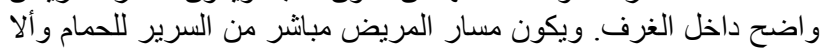
توضع قطع الأثاث علي طول هذا والثان المسار.

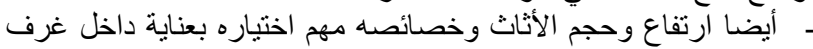

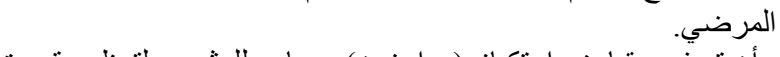

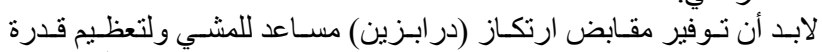

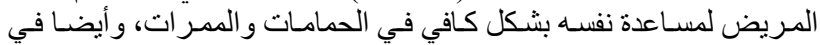

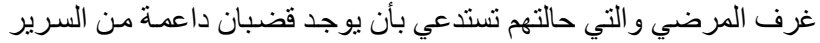

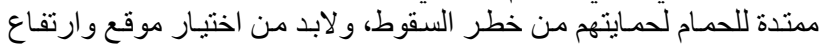

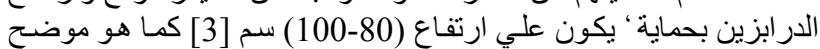

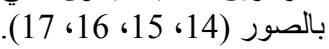
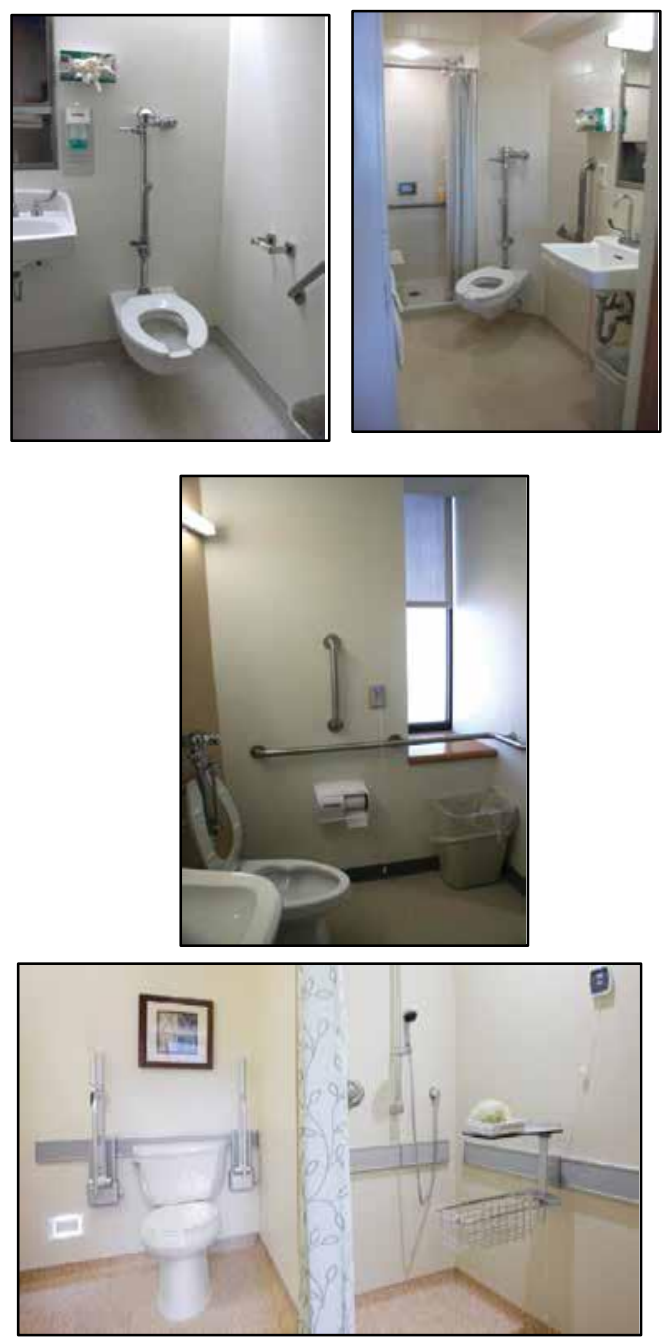

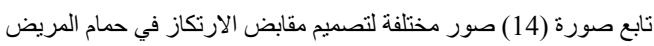

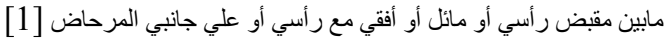


A: 26 HEBA MOHAMED AHMED MOHAMED ABDU, SHERIF AHMED ALI SHETA AND WAEL SEDDIKBDELATIF MOUSTAFA

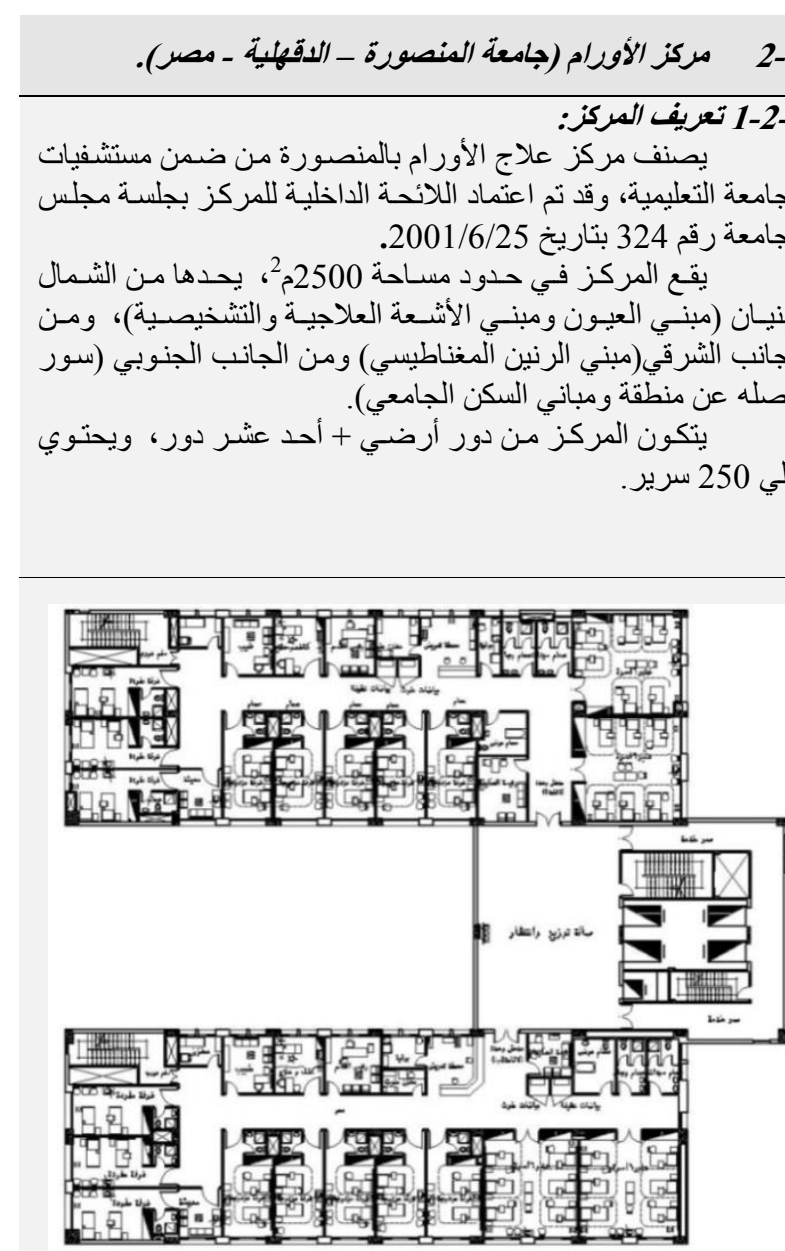

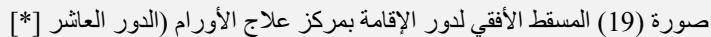

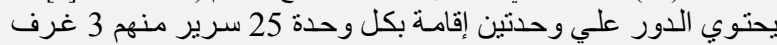

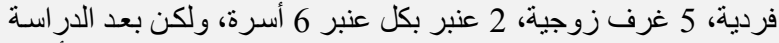

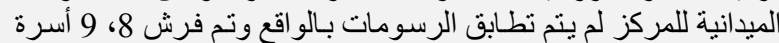

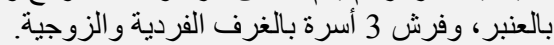

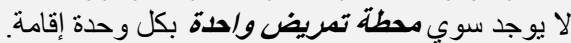

1-4 Rush University Medical Center Chicago, IL. 1-1-4 تعريف المركز:

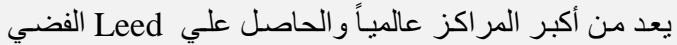

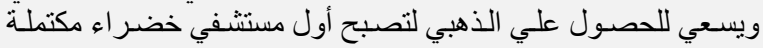

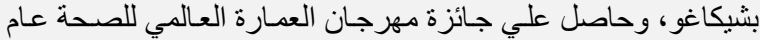
2013، ومعتمد من قبل لجنة مر افق التأهيل (CARF)

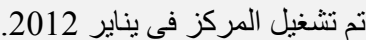

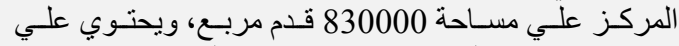

669غرفة مريض، جميع الغرف فردية، ويتميز بالكفة الكفاءة في استخدام

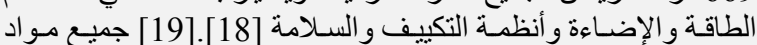

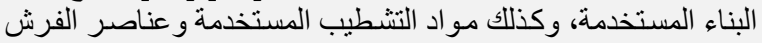
جميعا معاد تدوير ها [22].

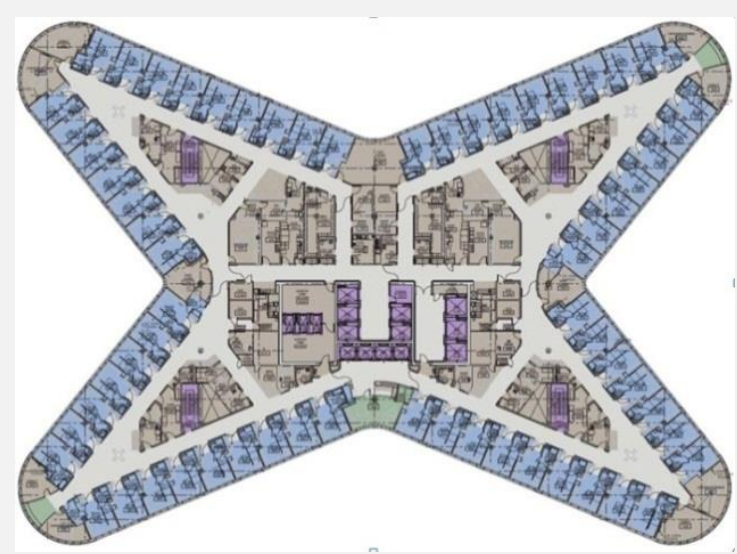

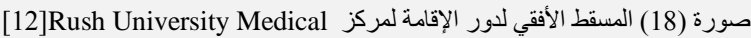

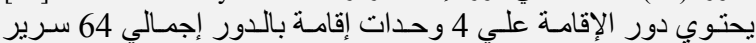
(كل وحدة 16 سرير ).

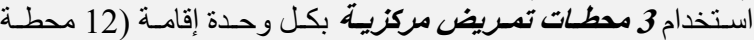

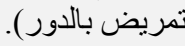
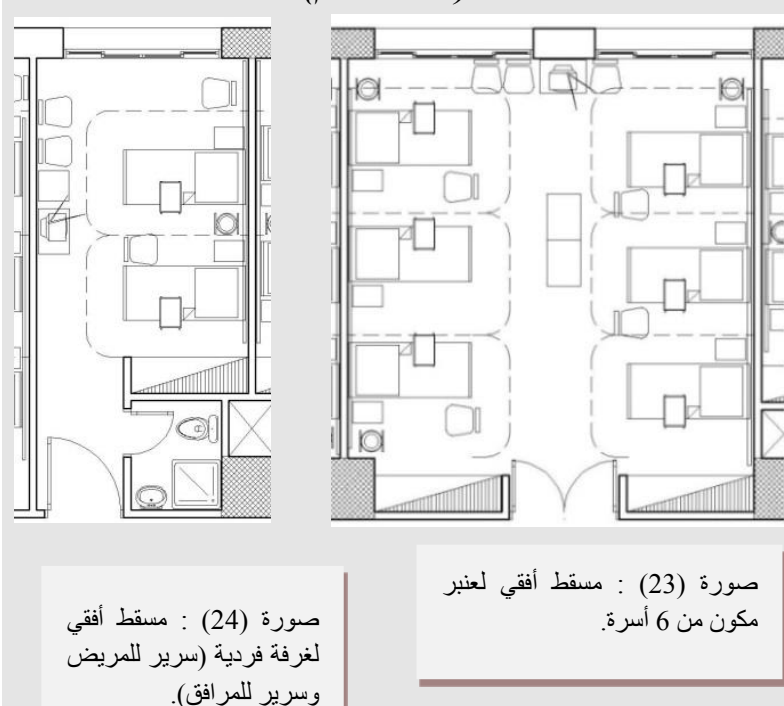
2-1-4 تحليل غرفة المريض (ألمركز الطبي)

\section{4}

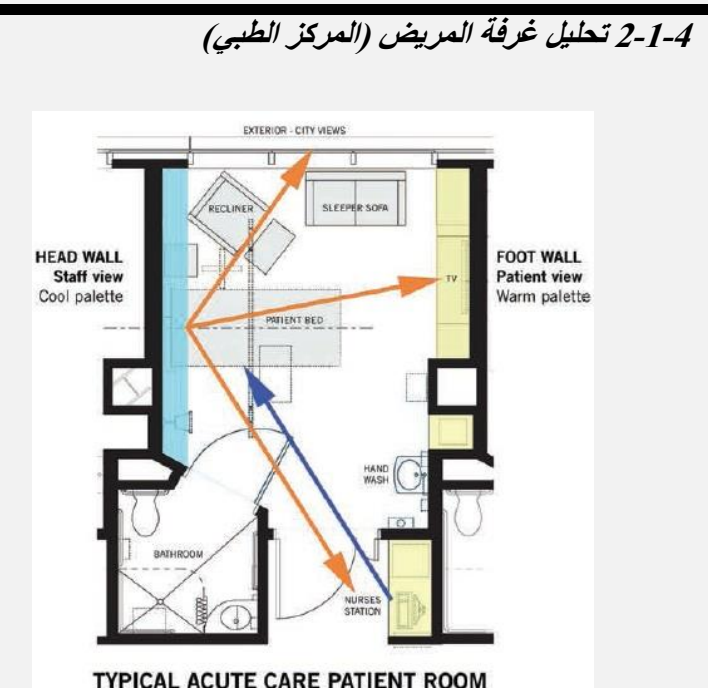

صورة (20) المسقط الأفقي لغرفة المريض بالمركز 


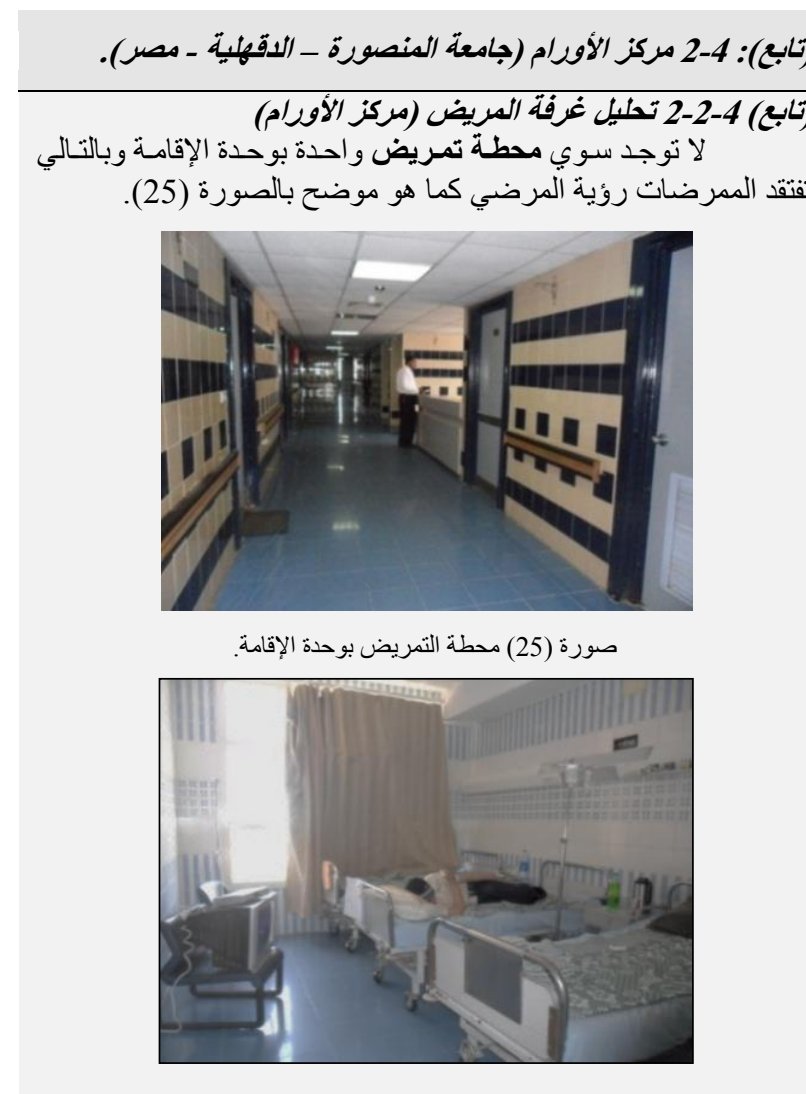

صورة (26) غرفة المريض الفردية وتحتوي علي 3 أسرة مرضي بالو اقع.

تكوين الغرفة Inboard head wall location وبالتالي الدا من الضوضاء الصنادر من الممر.

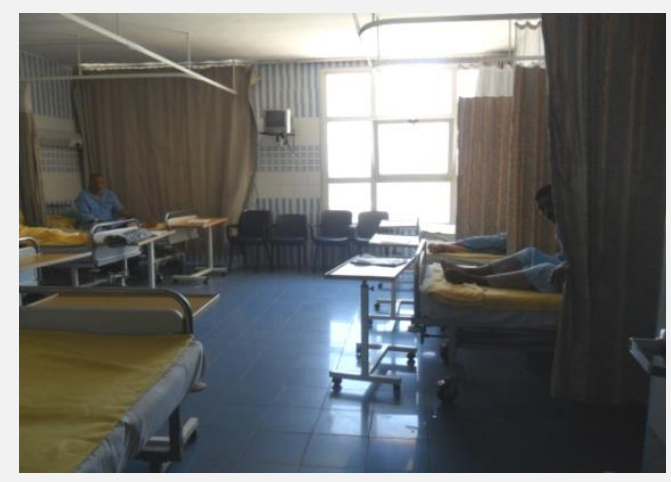

صورة (29) عنبر المرضي ويحتوي علي 9 أسرة بالواقع.

عدم وضوح رؤية باب العمام، وسهولة وصول المريض إليه

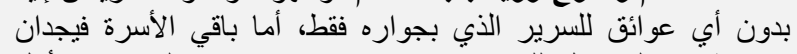

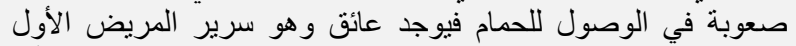

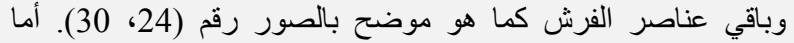

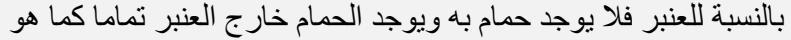

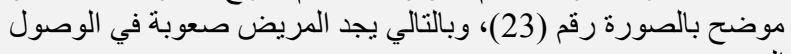

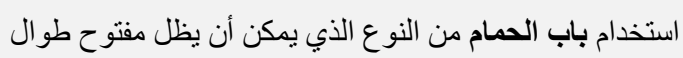

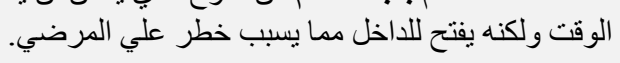

(تابع) : 1-4 المركز الطبي بجامعة راش بشيكاغو. Rush University Medical Center Chicago, IL. (تابع) 2-1-4 تحليل غرفة الهربض (المركز الطبي)

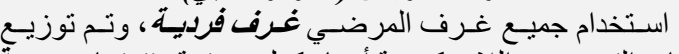

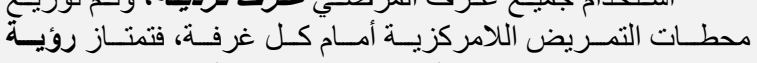

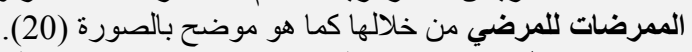

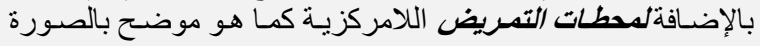

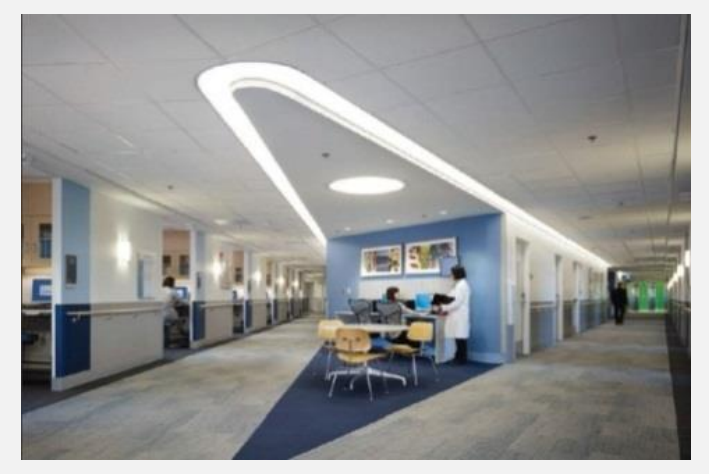

صورة (21) محطة التمريض المركزية بوحدة الإقامة.

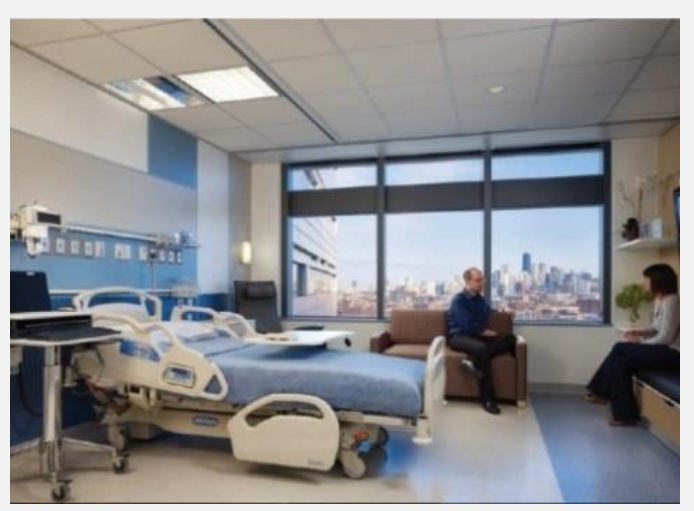

صورة (22) غرفة المريض بالمركز [20] [29] [22]

تكوين الغرفة Inboard head wall location وبالتالي الحد من الضوضاء الصنادر من المر.

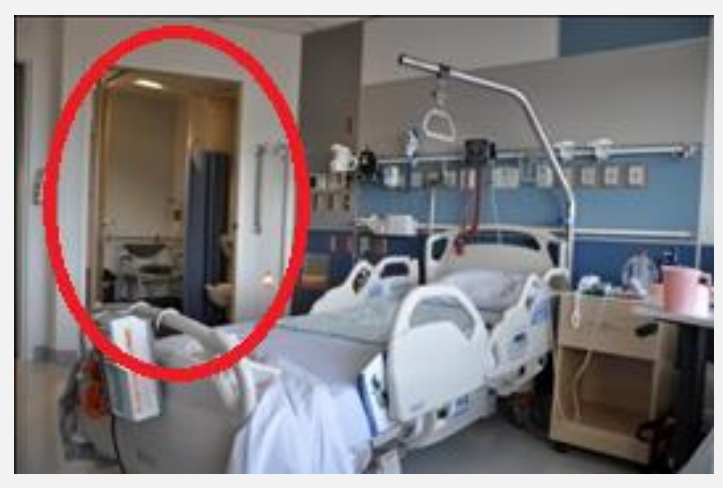

صورة (27) غرفة المريض بالمركز [23]

وضوح رؤية باب العمام وسهولة وصول المريض إليه

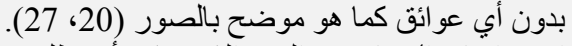

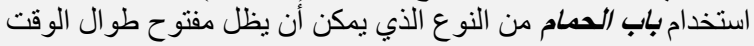
واتجاه فتح الباب للخار جما كما هو موضح بالصون الصورة (27). 
A: 28 HEBA MOHAMED AHMED MOHAMED ABDU, SHERIF AHMED ALI SHETA AND WAEL SEDDIKBDELATIF MOUSTAFA

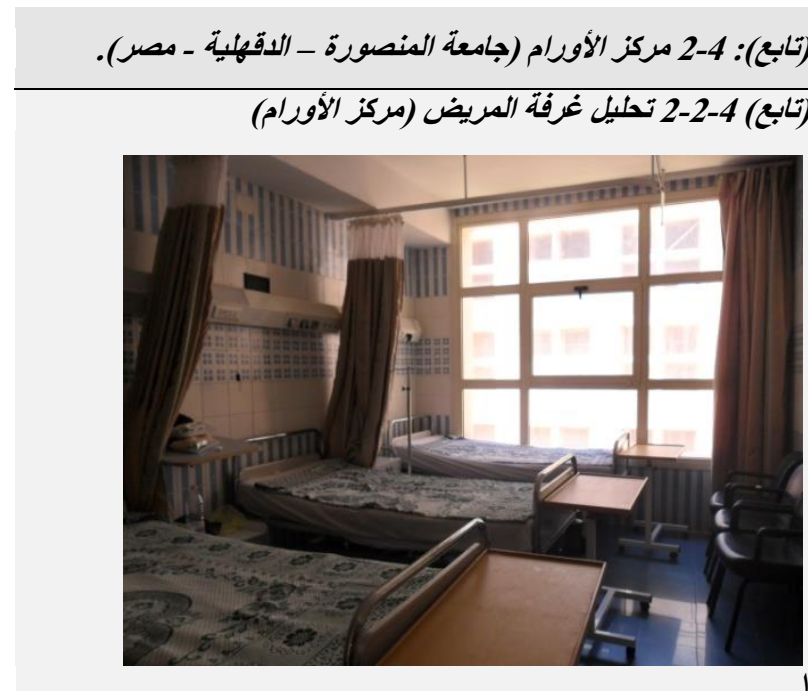

صورة (30) غرفة المريض الزوجية بالو اقع بها 3 أسرة.

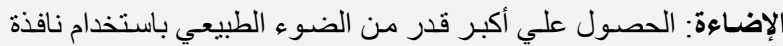
بعرض الغرفة كما هو موضح بالصورة الصبرة من رقم (30).

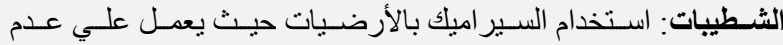

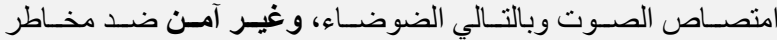

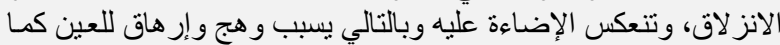

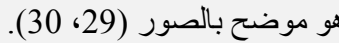

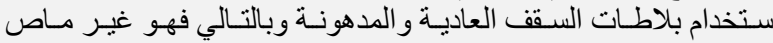

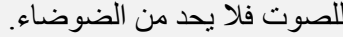

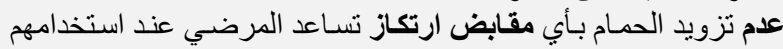

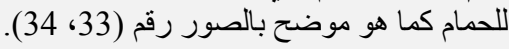
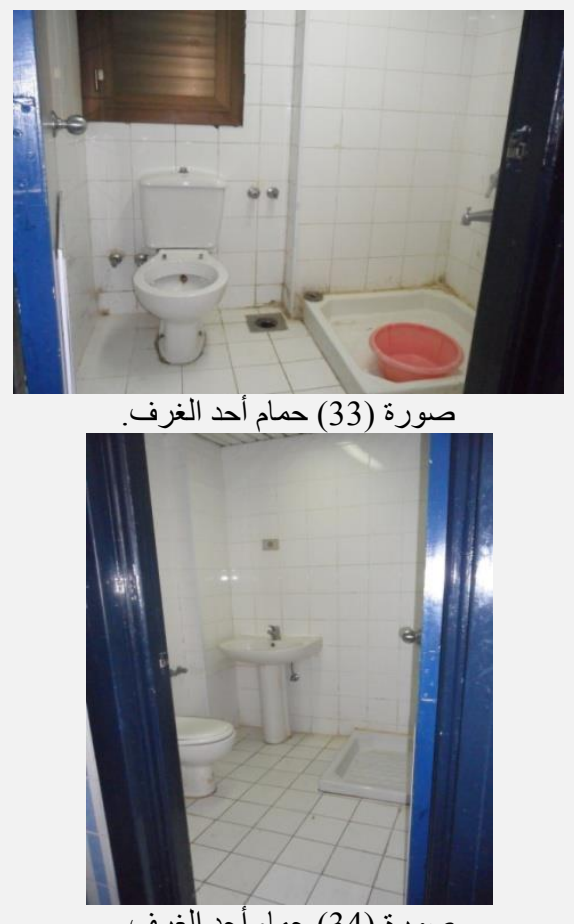

تزويد الحو ائط بمقابض ارتكاز تسـاعد المرضـي عن الحركة بـالممرات أمام الغرف كما هو موضح بالصورة رقم (35).
(تابع) : 1-4 المركز الطبي بجامعة راش بشيكاغو. Rush University Medical Center Chicago, IL (تابع) 4-1-2 تحليل غرفة المريض (المركز الطبي)

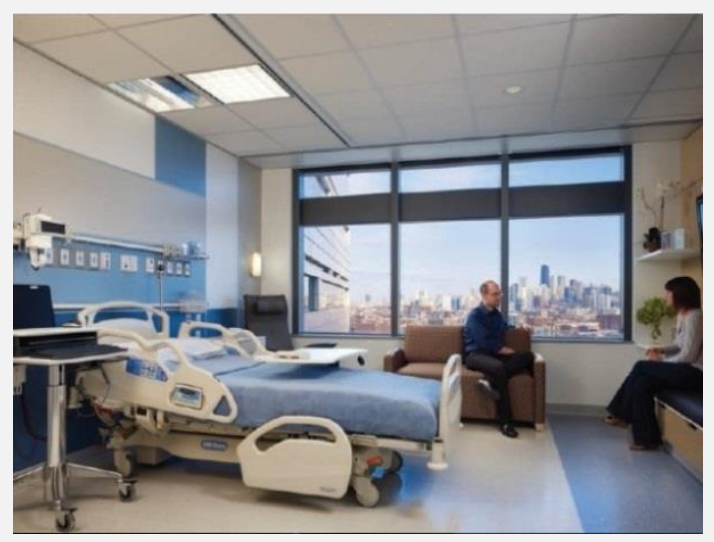

صورة (28) غرفة المريض بالمركز [20] [21] [22]

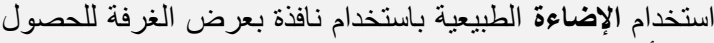

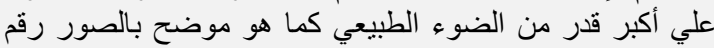

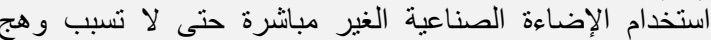

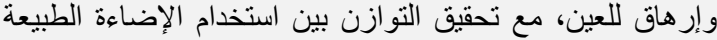

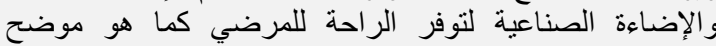

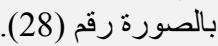

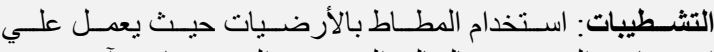

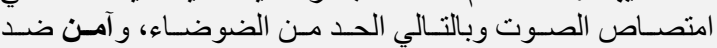

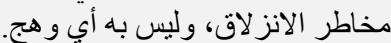

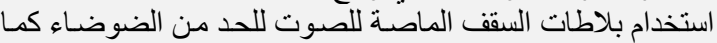
هو موضح بالصورة (28). تزويد الحمام بمقابض ارتكاز بعرض المر الحور ائط و عند مدخله و علي

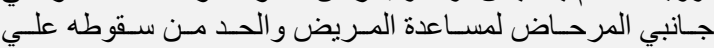

الأرض كما هو موضح بالصورة رقة لمنرة (23).

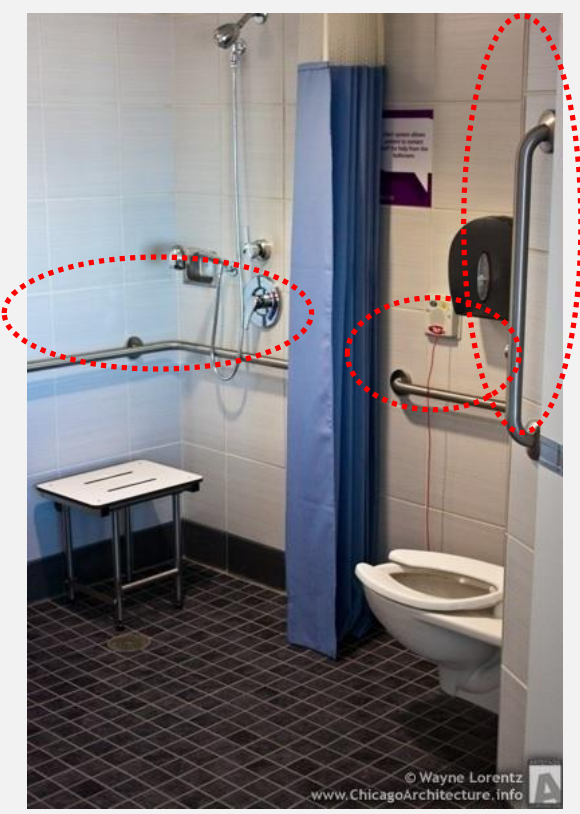

صورة (31) حمام غرفة المريض [24]

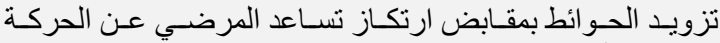

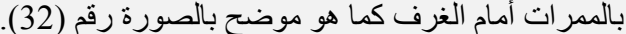




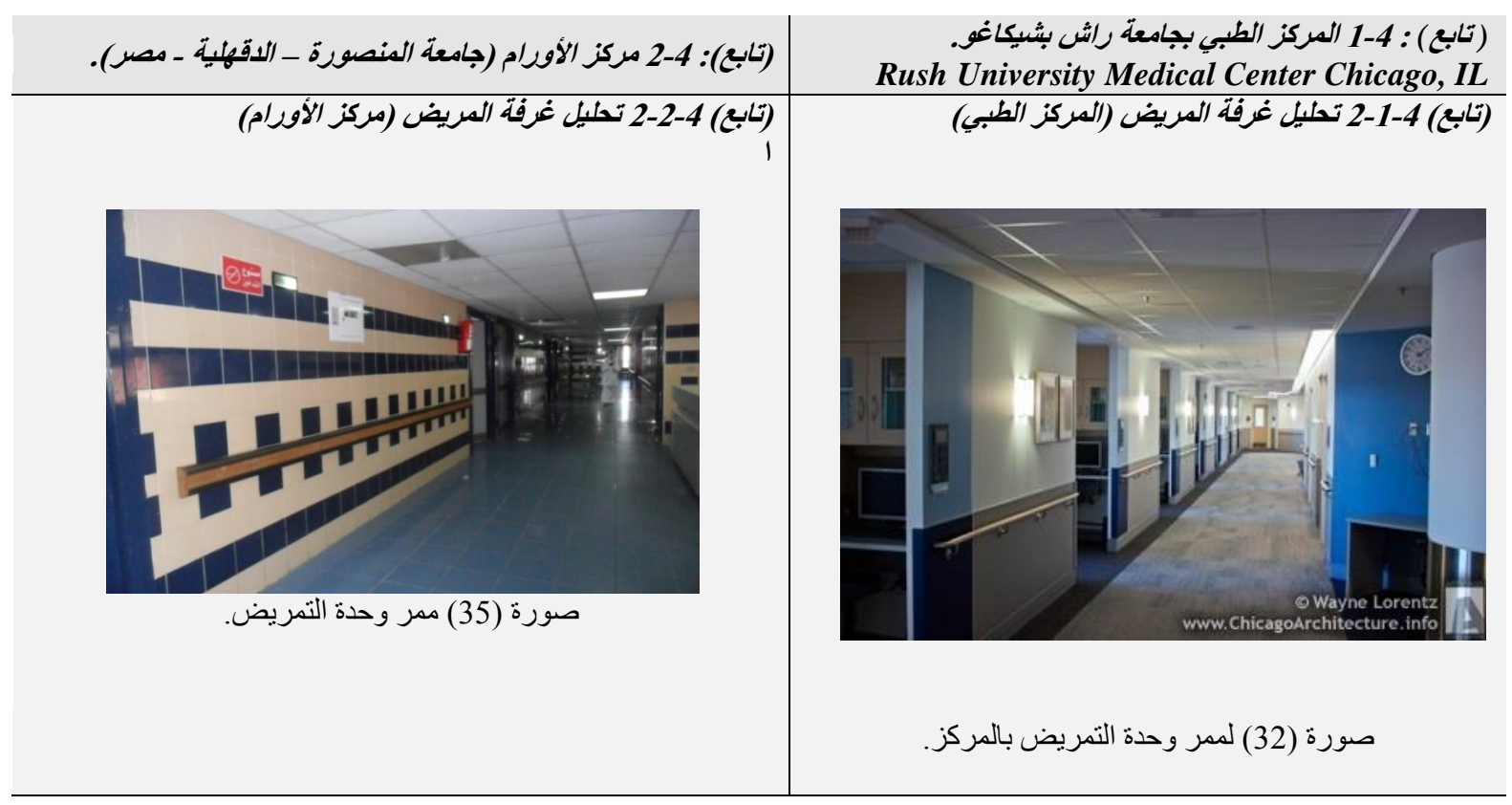

تابع جدول (4) تحليل مقارن بين المركز الطبي بجامعة راش وبين مركز أورام المنصورة

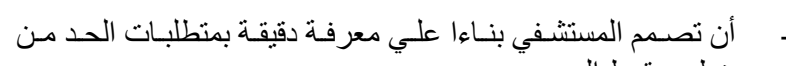
خطر سقوط المرضي.

تجديد مستشفيات مصر العرضر القائمة وتطبيق تللك المتطلبات عليها للحد من خطر سقوط المرضي.

إعادة النظر في مفهوم جودودة المستشفيات و والاهتمـام بـالجودة عمليا

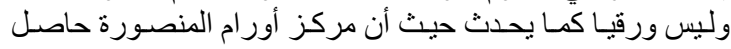
علي شهادة الجودة.

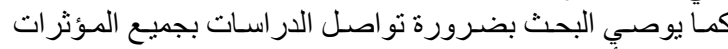

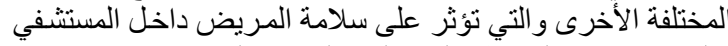

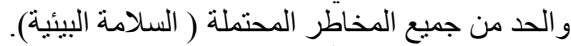

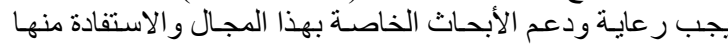

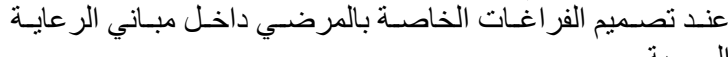

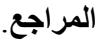

[1] Calkins, Margaret p. \& Biddle, Stacey \& Biesan, Orion (2012), "Contribution of the Designed Environment to Fall Risk in Hospitals", the Center for Health Design, Ideas Institute :P. 12, .P.33, $14,13,35,34$

[2] Dalke, Hilary\& Littlefair, Paul. J\& Loe, David. L and Camgoz. N (March 2013), "Lighting and Colour for Hospital Design", London South Bank University, NHS Estates, TSO, London. P. 49

[3] Gulwaodi, Gowri Betrabet \& Calkins, Margaret p. (2008), "The Impact of Healthcare Environmental Design on Patient Falls", The center for Health Design, The United States of America. P11,12, 15

[4] Hardy, Jeff (2006), "Patient Safety \& Quality Healthcare, Facility Design: No Hidden Patient", www.psqh.com..P 23, 24,25,22

[5] Inspiring the Building Team (October 2009), "Building Design+ Construction: RightSizing Healthcare", Volume 50, No.10, www.BDCnetwork.com.. P. 44

[6] Joseph, Anjali and Ulrich, Roger (2007), "Sound Control for Improved Outcomes in Healthcare Settings", The Center for Health

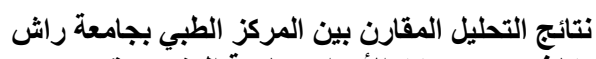

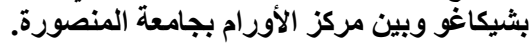

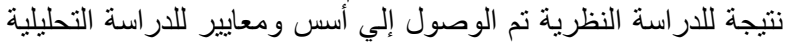

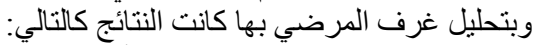

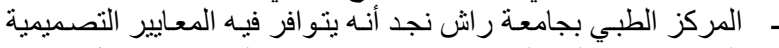

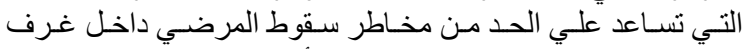

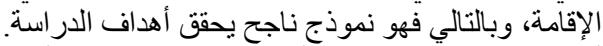

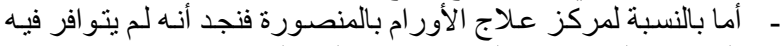

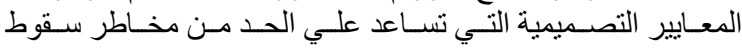

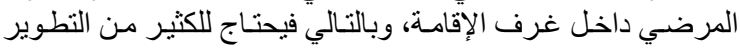

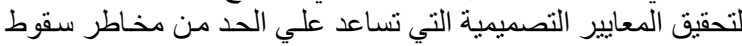

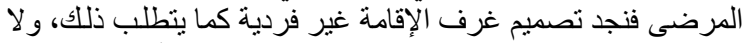

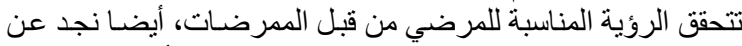

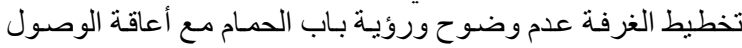

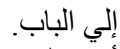

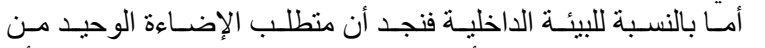

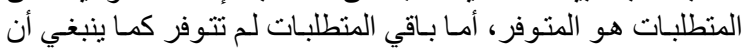
تكون عليه للحد من خطر سقوط المرضي. أمرضي.

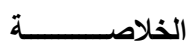

تتمثل المشكلة في وجود فجوة بين عملية التصميم وبين تطبيق

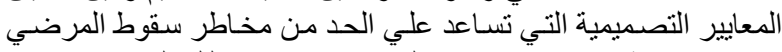

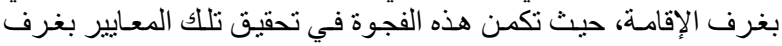

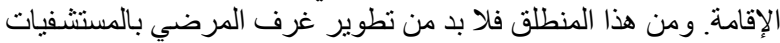

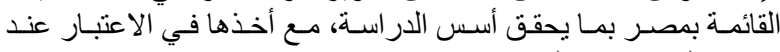
تصميم المستشفيات الجديدة.

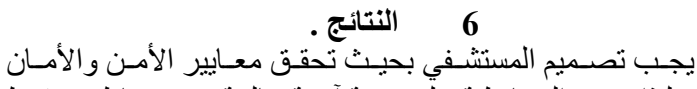

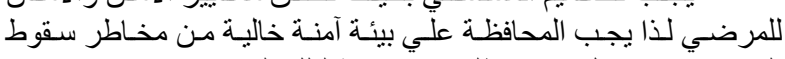

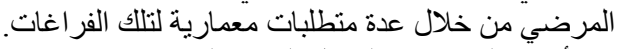

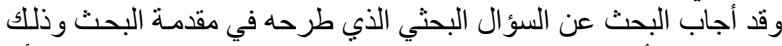

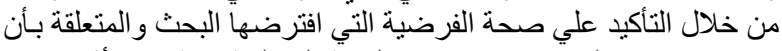

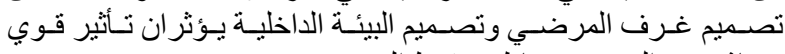
ومباشر في الحد من مخاطر سقوط المرضي.

$$
7
$$

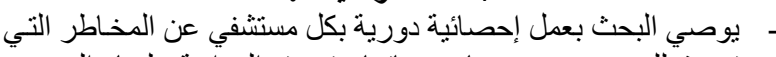

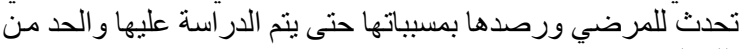


A: 30 HEBA MOHAMED AHMED MOHAMED ABDU, SHERIF AHMED ALI SHETA AND WAEL SEDDIKBDELATIF MOUSTAFA

[21] http://www.yelp.com/biz_photos/rushuniversity-medical-center-chicago-3 $\quad$ Nov 2015).

[22] http://www.rrgphotography.com/rush/ $\quad$ (Nov 2015).

[23] http://newsarchive.medill.northwestern.edu/chic ago/news-198227.html (Nov 2015).

[24] www.chicagoarchitecture.info (Nov 2015).

التحالف السكندري لسـامة المرضي APSA (نوفمبر 2015)،

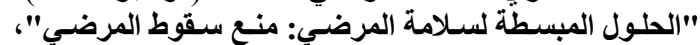

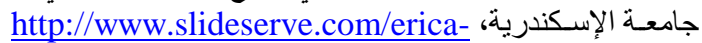
hardin/7016796

المركز القومي لبحسوث الإسـكان و البنـاء (2012)، "المعسايير

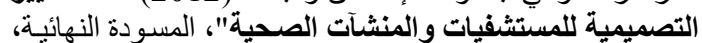

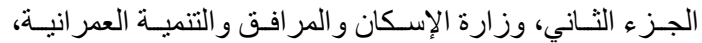

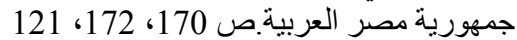

حمد الو هيب، عبد الحكيم (2007)، "مدية، صدئ توفر وتطبيق وسـائل

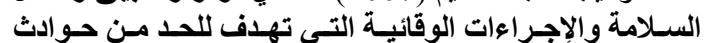

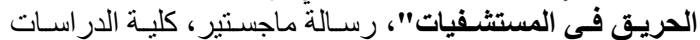

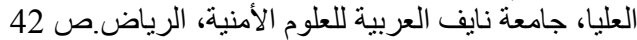

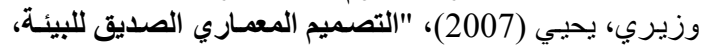
نحو عمارة خضر اءع"، الهيئة المصرية العامة للكتاب.
Design, Robert Wood Johnson Foundation, www.healthdesign.org.p2

[7] NHS Scotland (March 2014), "Health Building Note 00-01: General design guidance for healthcare buildings", Health Facilities Scotland, London, https://www.gov.uk/government/collections/heal th-building-notes-core-elements.p47

[8] NHS, Infection Prevention and control team (2012), "Infection Prevention and Control Guidance for new buildings and refurbishment of the existing environments", version $2, \quad \mathrm{p} 11$, https://www.lincolnshirecommunityhealthservic es.nhs.uk.

[9] Onaran, Bilge Sayil (February 2009), "Sustainable Therapy Room Surfaces in Acute Mental Health Hospital", Hacettepe University, Faculty of Fine Arts, Interior Architecture and Environmental Design Department, Turkey, Issue 2, volume 5.p225

[10] Phiri, Michael (2004), "One Patient One Room- Theory \& Practice: An evaluation of The Leeds Nuffield Hospital", A study report, School of Architecture, University of Sheffield.p54

[11] Swinney, Marlene and Iuss N and S task Group (2014), "Internal Wall Finishes in Healthcare Facilities",V1, National Department of Health, www.iussonline.co.za.p4

[12] Webb, Kenneth \& Buie, Allen, "Health care 101: Introduction to Medical- Surgical Patient Unit Design", The American Institute of Architects Academy of Architecture for Health,

http://www.aia.org/aiaucmp/groups/aia/documen ts/presentation/aiab086349.pdf(January 2015).

[13] Yee, Roger (2006), "Healthcare Spaces 3", New York, Visual Reference Publications, INC. P. 16,102

[14] http://www.healthcaredesignmagazine.com/artic le/using-evidence-based-strategies-design-safeefficient-and-adaptable-patient-rooms (January 2015).

[15] http://www.healthcaredesignmagazine.com/artic le/dublin-methodist-hospital-applying-evidencebased-design-race-revolutionize-healthcare (Nov 2015).

[16] http://www.nbbj.com/work/miami-valleyhospital-heart-and-orthopedic-center/ (October 2015).

[17] http://www.rtkl.com/projects/cadence-healthnow-northwestern-medicine-cadence-hospitalbed-pavillion/ (nov 2015)

[18] http://health.usnews.com/besthospitals/area/il/rush-university-medical-center6430985 (Nov 2015).

[19] http://www.alucobondusa.com/blog/alucobondplus-helps-rush-university-medical-centertower-transform-chicago (Nov 2015).

[20] http://aidaarchitecture.blogspot.com.eg/2015/10/rushuniversity-medical-center-by.html (Nov 2015). 